Introduction: Protein phosphatases (PP) and kinases are known to regulate the cell cycle dynamics. Although kinases have been studied extensively, most of the phosphatases are still unexplored. Therefore, the present study aimed to investigate the association of an isoform of PP1 family protein phosphatases 1 gamma 2 (PP1 $\gamma 2$ ) in the regulation of cervical cancer HeLa cell proliferation.

Material and methods: Expression of PP1 2 transcript and protein was assessed in the cervical cancer cell line of HeLa cells through RT-PCR and western blotting. Flow cytometry was employed to confirm its expression quantitatively, and Immuno-fluorescence was done to evaluate the distribution of PP1 $\gamma 2$ in the dividing mononuclear and Taxol-induced multipolar HeLa cells. PP1 1 2-specific siRNA-mediated silencing was done to understand downstream pathways. The effect of the hypoxic tumour microenvironment on PP1 $\gamma 2$ expression was also evaluated.

Results: RT-PCR and western blotting confirmed the expression of PP $1 \gamma 2$ in HeLa cells, and flow cytometry analysis established intracellular expression of PP1 $\gamma 2$. Immunofluorescence is localized PP1 2 in the nucleus of mononuclear cells during interphase, whereas it is transiently redistributed to spindle poles throughout the cell division and localized back to the nucleus after complete karyokinesis. Taxol-induced multipolar HeLa cells also showed a temporal redistribution of PP1 $\gamma 2$ on the spindle poles. Hypoxic conditions upregulated PP1 $\gamma 2$ expression, but downregulated PP1 $\gamma 2$ levels through siRNA increased GSK3 $\beta$ phosphorylation.

Conclusions: Collectively, data suggests that PP1 $\gamma 2$ is modulated during HeLa cell division and regulates GSK3 $\beta$ phosphorylation, which may regulate downstream signalling of cell division.

Key words: cell cycle, cervical cancer, protein phosphatases, cervical cancer diagnosis.

Contemp oncol (Pozn) 2021; 25 (2): 125-132 DOI: https://doi.org/10.5114/wo.2021.107745

\section{Modulation of protein phosphatase 1 gamma 2 during cell division of cervical cancer HeLa cells}

\author{
Saurabh Kumar Agnihotri ${ }^{1 *}$, Parmita Kar $^{2 *}$, Manish Singh ${ }^{3}$, Garima Pant ${ }^{3}$, \\ Kalyan Mitra ${ }^{3,4}$, Madan Lal Brahma Bhatt ${ }^{1}$, Monika Sachdev ${ }^{2,4}$
}

'Department of Radiotherapy, King George's Medical University, Lucknow 226 003, India 2Endocrinology Division, CSIR-Central Drug Research Institute, Lucknow 226 031, India ${ }^{3}$ Sophisticated Analytical Instrument Facility, CSIR-Central Drug Research Institute, Lucknow 226 031, India

${ }^{4}$ Academy of Scientific and Innovative Research (AcSIR), Ghaziabad 201 002, India

*These authors contributed equally to this work.

\section{Introduction}

Protein phosphatases (PP) and kinase are critical for the regulation of many biological functions [1]. Protein phosphatase 1 (PP1) is a member of the serine/threonine phosphatases family and has been reported as highly conserved among eukaryotes [2]. In the cellular system, the nuclear region has been reported as a highly dynamic compartment, and phosphorylation of protein plays an essential regulatory role in signalling pathways, including apoptosis [3]. Earlier reports revealed enriched PP1 activity in the cell's nucleus and crucial roles associated with the segregation of chromosomes. The localization patterns of PP1-isoforms are dynamic, and their distribution keeps changing throughout the cell cycle and in response to other cellular distresses [4]. The significance and role of the different PP1 isoforms and their association with mitotic events remain unclear, but they have distinct subcellular localization [5, 6].

Recent reports also indicate that PP1 [7] and PP2A [8] are required for mitotic exit. The PP1 isoforms play a crucial role in mitosis in mammals based on their localization pattern in the nucleus in G1 and $S$ phase cells [2]. PP1 consists of 4 isoforms: PP1 $\alpha, \mathrm{PP} 1 \beta / \delta$, PP $1 \gamma 1$, and PP1 $\gamma 2$. While PP1 $1 \gamma 1$ expresses ubiquitously, PP1 $\gamma 2$ is sperm-specific and essential for spermatogenesis [9]. PP1 $\alpha$ is localized to the centrosome, PP1 $\gamma 1$ is associated with the mitotic spindle's microtubules, and PP1 $\delta$ is strongly associated with chromosomes during mitosis [5]. PP1 $\gamma 1$ has been reported to play an essential role in cell cycle regulation, and its localization pattern has been correlated with the various stages of the cell cycle [10]. PP1 $\gamma 1$ and PP1 $\gamma 2$ are alternative splice variants, coded by [11] the PPP1CC gene. The PPP1CC gene is known to be associated with many biological processes including regulation of circadian rhythm [12] as well as cell cycle and cell division, glycogen metabolic process, and neuronal differentiation through protein dephosphorylation. Both the isoforms are identical in all respects, except that PP1 $\gamma 2$ has a unique 23-amino-acid carboxyl-terminal extension coded by an extra exon at the 3' terminal of this gene. So far, the expression of the PP1 $\gamma 2$ isoform has been reported to be testis-specific and plays a critical role in sperm maturation [9]. Simultaneously, several reports have reported that massive expression of germ cell-specific antigens (proteins) is a hallmark of cancerous cells [13]. However, the role of the PP1 22 isoform concerning cell cycle regulation or other key biological processes is not clear and remains unexplored. Hence, this study was designed to observe the profile of PP1 $\gamma 2$ in the cervical cancer cell line of HeLa cells. In vitro experiments were conducted to better understand the role of PP1 $\gamma 2$ isoform in the dynamics of the cell cycle transition associated with tumourigenesis. 


\section{Material and methods}

\section{Maintenance of cell line}

HeLa cells were obtained from ATCC (Manassas, VA) and maintained in the lab as per the standard protocol. Briefly, the cells were cultured in Eagle's Minimal Essential Medium (EMEM, M0894, Sigma Aldrich) supplemented with 10\% Foetal Bovine Serum (10082147, Gibco) and 1X antibiotic solution (15140122, Gibco) and kept at $37^{\circ} \mathrm{C}$ in $5 \% \mathrm{CO}_{2}$ in an incubator for growth and proliferation. The media was changed every $2-3$ days until the required 80-90\% confluence was obtained for the cells' sub-culturing.

\section{RNA isolation and RT-PCR}

Total RNA isolation from cells was done using tri reagent (T9424, Sigma Aldrich) as per the manufacturer's instruction [14]. cDNA synthesis was done with $2 \mu \mathrm{g}$ of RNA by using a GeneSure First CDNA synthesis kit (PGK162B, Puregene). PP1 2-specific (NCBI Reference Sequence NM_001244974.2) forward 5'GTGGTTGAAGATGGATATGA3' and reverse 5'CTGATGCAACCCTTG3' primers were used with Master Mix (K0171, Fermentas) as per the manufacturer's instructions. A PCR protocol consisting of an initial denaturation at $94^{\circ} \mathrm{C}$ for $2 \mathrm{~min}, 30$ cycles of denaturation for 30 secs at $94^{\circ} \mathrm{C}$, annealing at $43^{\circ} \mathrm{C}$ for 30 secs, extension for $1 \mathrm{~min}$ at $72^{\circ} \mathrm{C}$, and a final extension at $72^{\circ} \mathrm{C}$ for 5 min was used for PP1 2. Glyceraldehyde 3-phosphate dehydrogenase (GAPDH) (NCBI Reference Sequence NM_001256799.3) served as an internal control using 5'CACCATCTTCCAGGAGCGAG3' and 5'TCACGCCACAGTTTCCCGGA3' primers with an initial denaturation for 2 min at $94^{\circ} \mathrm{C}$, followed by 30 cycles of denaturation at $94^{\circ} \mathrm{C}$ for $30 \mathrm{sec}$ and annealing for $30 \mathrm{sec}$ at $54^{\circ} \mathrm{C}$, extension at $72^{\circ} \mathrm{C}$ for $1 \mathrm{~min}$, and a final extension for $5 \mathrm{~min}$ at $72^{\circ} \mathrm{C}$. Commercially obtained Human Testis RNA (540049, Stratagene) was used as a positive control for PP1 2 amplification. The products were run on a $2 \%$ agarose gel for confirmation of product size.

\section{Protein extraction and quantification}

Protein extraction from whole cells was done using RIPA buffer (R0278, Sigma Aldrich) as per the manufacturer's instructions. Briefly, $100 \mu \mathrm{L}$ of RIPA buffer containing $1 X$ protease (p8340, Sigma Aldrich) and phosphatase inhibitor (p0044, Sigma Aldrich) was added per $10^{6}$ cells. After lysis, cells were centrifuged at $14000^{*} \mathrm{~g}$ for 10 minutes at $4^{\circ} \mathrm{C}$ to aspirate the protein-containing supernatant. The NE-PER kit was used (78833, Thermo Scientific) for protein extraction from cytoplasmic and nuclear fractions as per the manufacturer's instructions. Protein quantification was done by the Bradford method [15] using Bradford Reagent (B6916, Sigma Aldrich).

\section{Specificity of the anti-PP $1 \gamma 2$ antibody}

PP1 22 is a 337 amino acid protein with a molecular mass of around $39 \mathrm{kDa}$. PP1 $1 \gamma 1$ and PP1 $\gamma 2$ isoforms are coded as 2 different splice variants of the same gene PPP1CC. These isoforms are identical except a specific stretch of 23 amino acids at the C-terminal of the PP1 $\gamma 2$ isoform. Therefore, the full-length PP1 $\gamma 2$ protein cannot be explored to get PP1 $\gamma 2$-specific antibodies, and hence a synthetic peptide of only specific stretch of 23 amino acids (VASGLNPSIQKASNYRNNTVLYE) of PP1 $\gamma 2$ was used as an immunogen to obtain affinity-purified anti-PP1 $\gamma 2$-specific antibodies in Rabbit.

\section{Western blotting}

Proteins were resolved through SDS-PAGE. Briefly, 25-50 $\mathrm{\mu g}$ of protein lysate was mixed with $2 X$ Laemilli buffers containing $\beta$-mercaptoethanol (M6250, Sigma Aldrich), and electrophoresis was performed at a constant voltage (50 V). Proteins were electroblotted on to a nitrocellulose membrane (66485, Pall Corporation) using a semi-dry transfer system (Pierce Power Station, Thermo Scientific), and the membrane was blocked in $0.1 \%$ Tween-20 added in phosphate buffer saline (PBST) containing 2\% Bovine Serum Albumin (BSA) for $1 \mathrm{hr}$ at room temperature $\left(25^{\circ} \mathrm{C}\right)$. The membrane was incubated with the anti-PP1 $\gamma 2$ antibody (1:3000) overnight (12 hrs) at $4^{\circ} \mathrm{C}$. After washing, the membrane was incubated with the HRP-conjugated secondary antibody $(1: 10,000)$ for 2 hrs at room temperature $\left(25^{\circ} \mathrm{C}\right)$. Again, after washing, the blot was developed with Immobilon Western Chemiluminescent HRP Substrate (WBKLSO500, Millipore), and a chemiluminescent signal was measured using ImageQuant ${ }^{\text {TM }}$ LAS 4000 gel doc system (GE). Commercially obtained normal human testis lysate (1313, ProSci) was loaded as the positive control.

\section{Flow cytometry analysis of HeLa cells}

Confluent cells were harvested and fixed by $4 \%$ paraformaldehyde. After washing with PBS, the cells were permeabilized using 0.1\% Triton X 100 and $0.05 \%$ NP-40 in $1 X$ PBS and washed with PBS. The cells were centrifuged at $300^{*} \mathrm{~g}$ for $3 \mathrm{~min}$ and incubated with anti-PP1 $\gamma 2$ antibody for $1 \mathrm{hr}$ on ice, followed by washing with PBS. Cells were then incubated with specific fluorescent-tagged secondary antibody for $1 \mathrm{hr}$ on ice in the dark. After washing, the cells were analysed on a BD-FACS-Aria along with all the required controls.

\section{Taxol treatment in HeLa cells}

Around 0.1 million cells/well were seeded in 2-chamber polystyrene tissue culture glass slides (BD Biosciences, USA) and kept at $37^{\circ} \mathrm{C}$ in a $5 \% \mathrm{CO}_{2}$ incubator overnight. After the incubation, the media was replaced with a fresh EMEM medium containing 5, 10, 25, 50, and 75 nM Taxol (Paclitaxel, NAPROD lifescience) in replicates and cultured for an additional $2 \mathrm{hrs}$. These cells were then fixed with $4 \%$ paraformaldehyde for 15 minutes at $37^{\circ} \mathrm{C}$; after washing with PBS, the cells were processed for the immunofluorescence studies.

\section{Immunofluorescence and confocal microscopy}

HeLa cells were seeded on coverslips, fixed and permeabilized as described earlier, and then blocked with $1 \%$ BSA for 2 hrs at room temperature $\left(25^{\circ} \mathrm{C}\right)$ followed by incubation with PP1 22 specific primary antibody at a dilution of 1:250 and $\alpha$-Tubulin (32-2500, Invitrogen)-specific primary antibody at a dilution of 1:500 overnight (16 hrs) at $4^{\circ} \mathrm{C}$. 
After incubation, the cells were washed thrice with PBST and once with PBS, and then probed with polyclonal Cy3 $(\lambda$ ex/ $/$ em $=550 / 570 \mathrm{~nm})$-labelled AffiniPure Donkey Anti-Rabbit IgG secondary antibodies (cat\# 711-165-152, Jackson ImmunoResearch Laboratories, Inc.) at a dilution of 1:500 for PP1 $\gamma 2$ expression/localization experiments. FITC $(\lambda$ ex $/ \lambda$ em $=490 / 525 \mathrm{~nm})$-labelled anti-mouse for $\alpha$-Tubulin and $0.25 \%$ DAPI $(\lambda$ ex/ $/ \lambda$ em $=490 / 525 \mathrm{~nm})$ for the nuclear staining were used, and cells were incubated at $37^{\circ} \mathrm{C}$ in the dark for 2 hours at room temperature. The cells were rewashed as explained above, followed by mounting in Prolong Gold Antifade Reagent (P36934, Invitrogen) and visualized under a confocal laser scanning microscope (Carl Zeiss LSM 510 Meta) using a sequential mode of imaging using a Plan-Apochromat 63x/1.4 Oil DIC M27 objective.

For the red channel (PP1 $\gamma 2)$, Cy3 ( $\lambda$ ex/ $\lambda$ em $=555 / 569$ $\mathrm{nm}$ ) was excited using a $561 \mathrm{~nm}$ laser line (DPSS 561-610 laser), and the emission was captured using a BP: $575-615$ $\mathrm{nm}$ filter. For the green channel ( $\alpha$-Tubulin), FITC ( $\lambda$ ex/ $\lambda$ em $=490 / 525 \mathrm{~nm}$ ) was excited using a $488 \mathrm{~nm}$ laser line (Multi-line Argon laser) and the emission was captured using BP: $505-550 \mathrm{~nm}$ filters. For the blue channel (nucleus), DAPI $(\lambda$ ex $/ \lambda$ em $=358 / 461 \mathrm{~nm})$ was excited using $405 \mathrm{~nm}$ laser line (Blue Diode Laser) and the emission was captured using BP: $420-480 \mathrm{~nm}$ filters.

\section{Exposure of cervical cancer HeLa cells to a hypoxic environment}

For hypoxia experiments, cervical cancer HeLa cells were seeded on a $25 \mathrm{~cm}^{2}$ tissue culture flask until they reached $75 \%$ confluence and then exposed to either normoxic or hypoxic conditions for $24 \mathrm{hrs}$ and $48 \mathrm{hrs}$. In normoxic conditions the cells were maintained at $37^{\circ} \mathrm{C}$ in a humidified incubator with 5\% $\mathrm{CO}_{2} / 95 \%$ air. For hypoxia experiments, the culture medium was changed immediately before the cells were exposed to the hypoxic environment within the hypoxia chamber (STEMCELL Technologies, Cambridge, MA, USA), which was maintained at low oxygen tension $\left(1 \% \mathrm{O}_{2}, 5 \% \mathrm{CO}_{2}\right.$, and $\left.94 \% \mathrm{~N}_{2}\right)$. The treatment was initiated by introducing the culture in the hypoxia chamber and replacing the existing culture medium with deoxygenated medium. Deoxygenated medium was prepared before each experiment by equilibrating the medium with a hypoxic gas mixture containing $1 \%$ $\mathrm{O}_{2}, 5 \% \mathrm{CO}_{2}$, and $94 \% \mathrm{~N}_{2}$ at $37^{\circ} \mathrm{C}$. At the end of $24 \mathrm{hrs}$ and 48 hrs; no change in media colour was noted, indicating physiologic $\mathrm{pH}$ values between 7 and 8 were maintained throughout the experiment. After incubation of up to $48 \mathrm{hrs}$, cells were harvested, and the pellet was stored at $-20^{\circ} \mathrm{C}$ for further downstream experiments.

\section{SiRNA-mediated knockdown of PP1 $\gamma 2$}

Two siRNAs specific for PP1 $\gamma 2$ (Si1 sense GCCUGAACCCGUCCAUUCA, antisense UGAAUGGACGGGUUCAGGC Si2: sense CAUUCAGAAAGCUUCAAAU, antisense AUUUGAAGCUUUCUGAAUG and) were designed and commercially obtained along with a non-target scrambled one (SC). Around $0.3 \times 10^{6}$ cells were seeded in 6-well plates and incubated for 16-18 hrs.
At $60-70 \%$ confluence, siRNA transfection was done using lipofectamine 3000 (L3000-008, Life Technologies) as per the manufacturer's instructions. Briefly, $50 \mathrm{nM}$ of PP1 $\gamma 2$-specific siRNAs and scrambled siRNA were diluted in Opti-MEM medium (11058-021, Life Technologies). Lipofectamine 3000 was also diluted in Opti-MEM medium accordingly. The siRNA-lipid complex (lipoplex) was prepared by mixing the diluted siRNA and lipofectamine 3000 and added to the cells. Following incubation for 12 hrs at $37^{\circ} \mathrm{C}$ in $5 \% \mathrm{CO}_{2}$, exhausted media was replaced with a complete fresh medium.

\section{Statistical analysis}

The statistical analysis of the study was performed using the GraphPad Prism software (GraphPad, Version 5.00). Imaage (NIH) software was used for the densitometry analysis. Student's t-test for comparisons between 2 groups and one-way analysis of variance for multiple groups of data were used for the evaluation of statistical significance. Differences were considered statistically significant at a $p$ value $<0.05$, and results were represented as mean \pm SEM.

\section{Results}

\section{Expression of PP1 $\gamma 2$ in cervical cancer HeLa cells}

Expression of several germ cell-specific antigens have been reported in cancerous cells $[16,17]$, which can be explored as cancer biomarkers. Hence the expression of a testis-specific isoform of phosphatase PP1 $\gamma 2$ was explored in cervical cancer HeLa cells. Transcript expression of PP1 2 was confirmed through RT-PCR, which showed an amplicon of 205 bp specific for PP1 2 in HeLa cells. Similar results were shown by human testis mRNA, which was used as a positive control, and GAPDH amplification confirmed the integrity of mRNA (Fig. 1 A). A further signal of $\sim 39 \mathrm{kDa}$, specific for PP1 $\gamma 2$, was observed in HeLa cells as well as in the testis lysates in the western blot analysis (Fig. 1 B). Flow cytometry analysis of permeabilized HeLa cells also showed abundant intracellular expression of PP1 $\gamma 2$ (Fig. 1 C), whereas no signal was observed in un-permeabilized cells.

\section{Localization profile of PP1 $\gamma 2$ in cervical cancer HeLa cells}

Western blotting confirmed the abundant expression of PP1 22 in the cells' nuclear fraction, while cytoplasmic fraction showed a comparatively lower expression profile (Fig 2 A). The intensity of the whole cell lysate was higher compared to both the fractions through densitometry analysis (Fig 2 B). Expressions of $\beta$-actin and Histone-3 served as controls for the cytoplasmic and nuclear fraction, respectively. Indirect immunofluorescence through confocal microscopy also localized PP1 $\gamma 2$ predominantly in the HeLa cells' nuclear region, whereas discrete patches were observed in the cytoplasm. However, tubulin was found to be localized in the cytoplasm (Fig. 2 C).

\section{Modulation of PP $1 \gamma 2$ during mitotic transition}

To understand the modulation of PP1 $\gamma 2$ in cell cycle regulation, localization of PP1 $\gamma 2$ along with tubulin was evaluated in the various stages of dividing HeLa cells. PP1 2 was 
A
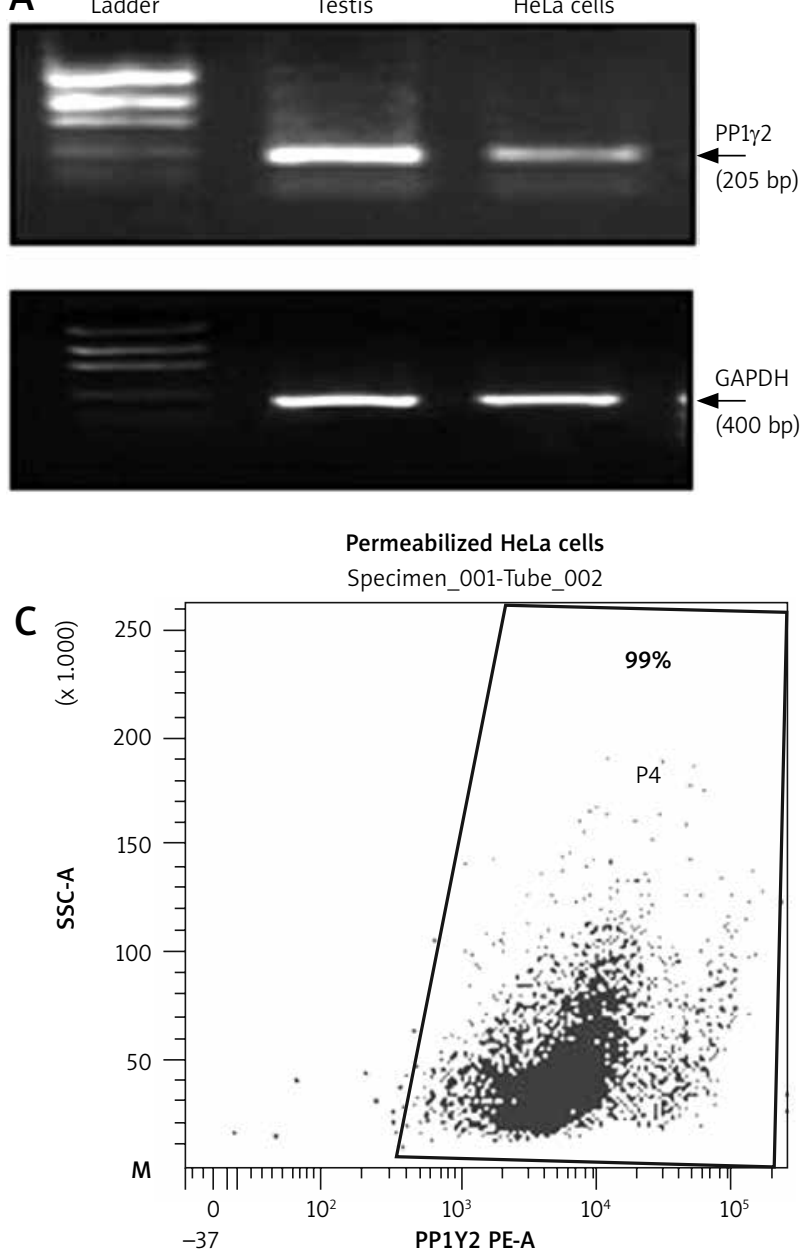

B
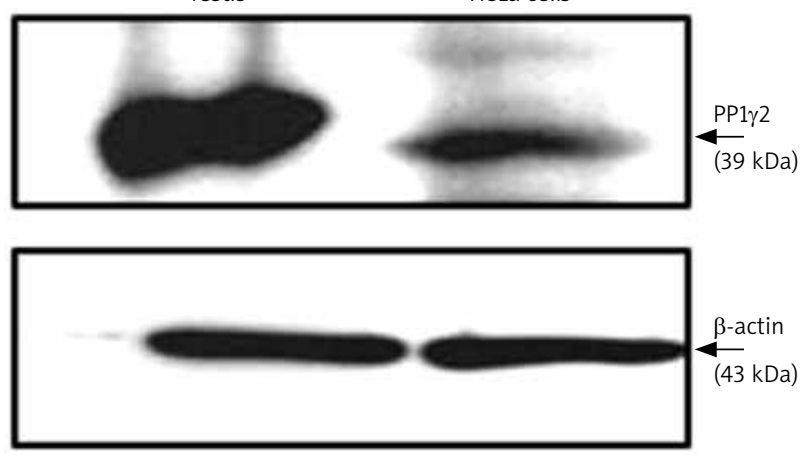

Un-permeabilized HeLa cells

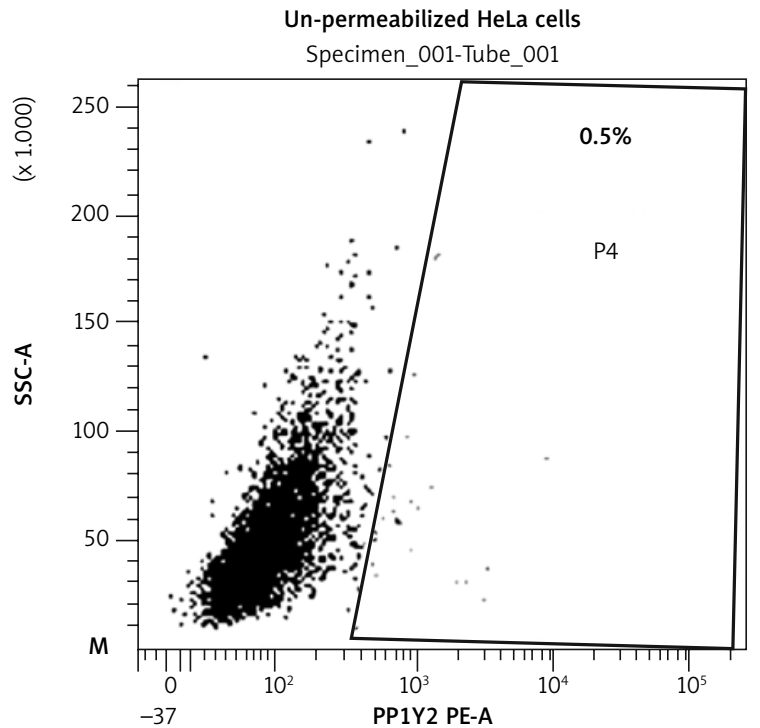

Fig. 1. Expression of protein phosphatases 1 gamma 2 (PP1 $\gamma 2)$ in cervical cancer HeLa cells. A - HeLa cells showed PP1 2 transcript ( 205 bp) through RT-PCR; testis extract was used as control for PP1 $\gamma 2$ while glyceraldehyde 3-phosphate dehydrogenase ( 400 bp) was used as an internal control, B - PP1 $\gamma 2$ protein $(\sim 39 \mathrm{kDa})$ expression was confirmed through western blot whereas $\beta$-actin $(\sim 43 \mathrm{kDa})$ was used as internal loading control, C - flow cytometry analysis of permeabilized live HeLa cells showed intense fluorescence of PP1 $\gamma 2$, whereas un-permeabilized cells did not show any fluorescence indicating predominant intracellular expression of PP1 $\gamma 2$

localized to the nucleus of the mononuclear cells, whereas signal of tubulin was noticed in the cytosol (Fig. 3 A-I). At the initiation of the cell division, PP1 $\gamma 2$ redistributed to the poles, whereas tubulin was localized to both the spindle poles and the cytosol (Fig. 3 A-II). In the mitotic phase, PP1 22 was found to be condensed and merged entirely with tubulin to the poles of the bipolar cells (Fig. 3 A-III). After complete cell division, PP1 22 re-localized again back to the nucleus even before the completion of cytokinesis; however, tubulin remained in the cytosol only (Fig. 3 A-IV) and did not overlay with PP1 $\gamma 2$.

Furthermore, to comprehend the dynamics of PP1 $\gamma 2$ during cell division, multinuclear cells were induced with Taxol. In the Taxol-induced multipolar cells, elevated expression of PP1 $\gamma 2$ merged completely with tubulin on multiple spindle poles, including tripolar (Fig. 3 B-I), tetrapolar (Fig. 3 B-II), and pentapolar cells (Fig. 3 B-III). Punctate staining of PP1 $\gamma 2$ was observed in the distorted chromosomal apparatus's vicinity, whereas tubulin remained located in the cytosol itself (Fig. 3 B-IV). These observations clearly indicate that PP1 22 has a specific pattern of modulation during the progression of the cell cycle.
PP1 $\gamma 2$ is upregulated during hypoxia in cervical cancer HeLa cells

Hypoxic conditions are known to be favourable for cancerous cells, but the exact rationale to make this environment favourable for cancerous cells remains unclear. Therefore, HeLa cells were grown under a hypoxic environment (1\% oxygen) and assessed for the expression of PP1 $\gamma 2$ through western blotting. Cells grown under a standard environment (normoxia, 21\% oxygen) were used as control. Interestingly, the results showed up-regulation of PP1 22 in hypoxic conditions, where upregulated levels of HIF1- $\alpha$ and $\alpha$-Enolases were used as the known markers of hypoxic conditions (Fig. 4 A). Densitometry analysis confirmed these results with statistical significance for PP1 $1 \gamma 2$ \& HIF1- $\alpha$, whereas upregulation of $\alpha$-Enolase was not statistically significant (Fig. 4 B).

\section{PP1 $\gamma 2$ silencing increases GSK3 $\beta$ phosphorylation}

It has been proven before that GSK3 $\beta$ is a direct substrate for PP1 $\gamma 2$ and dephosphorylates PGSK3 $\beta$ (Ser 9) to produce an active form of GSK3 $\beta$ [18]. An experiment was 
A

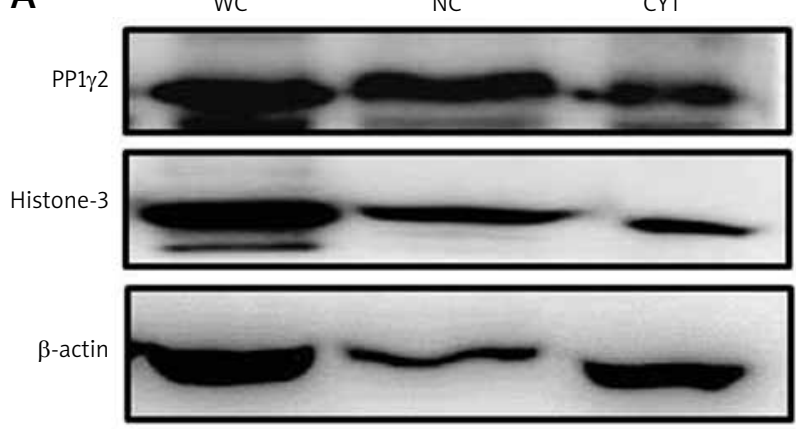

C
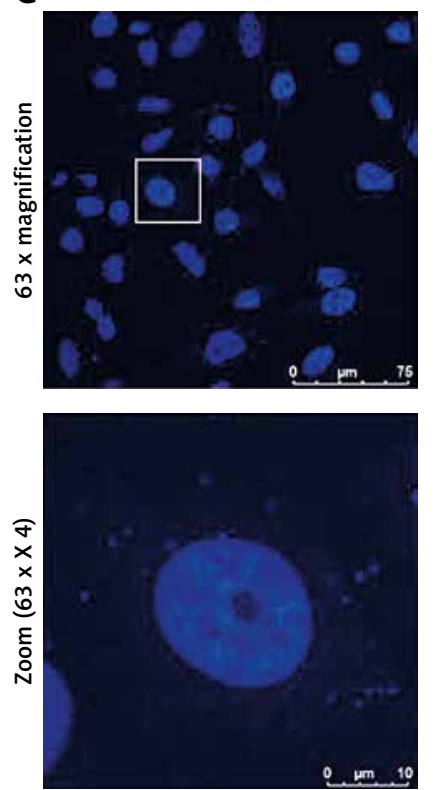

Tubulin (g)
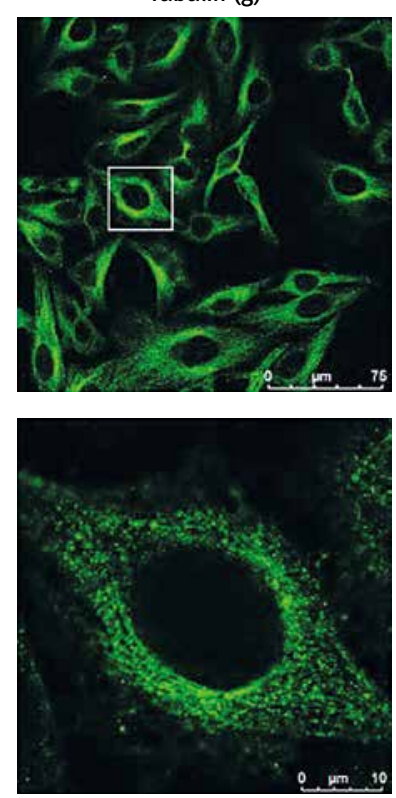

B

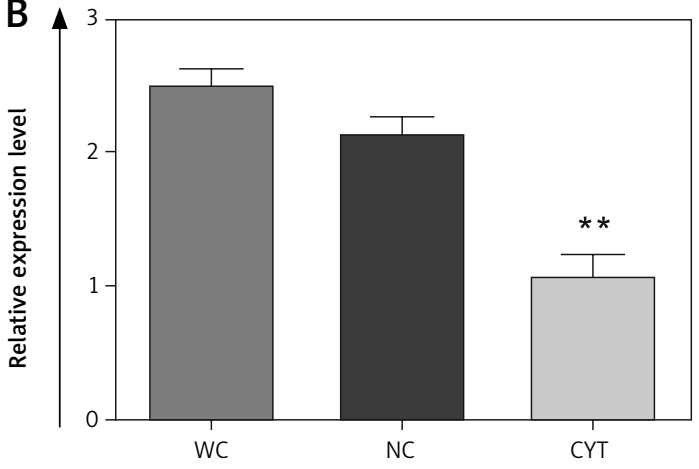

PP1 $\gamma 2(r)$
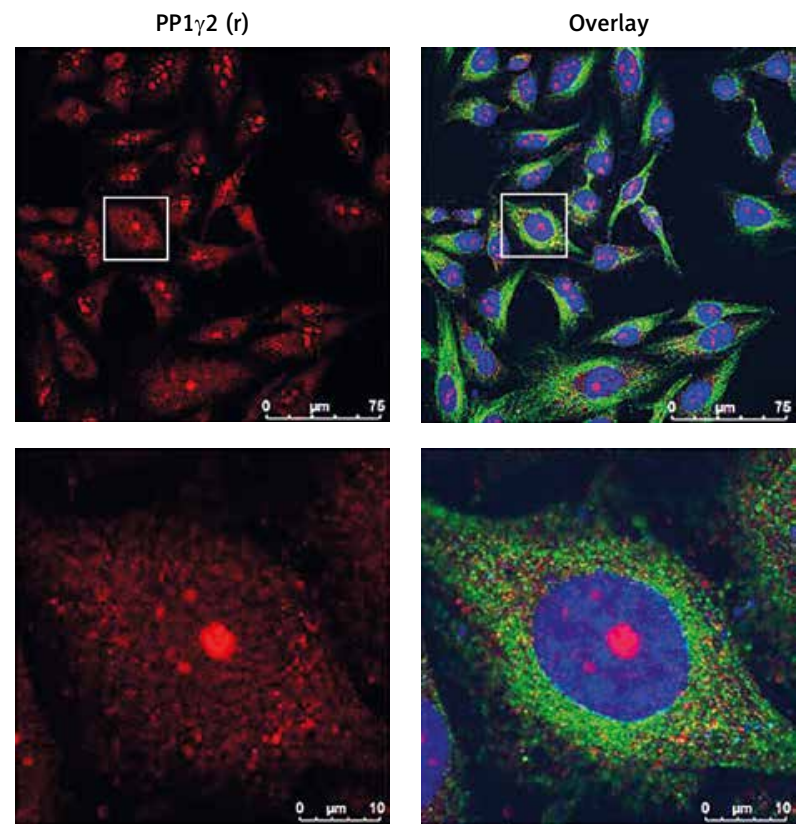

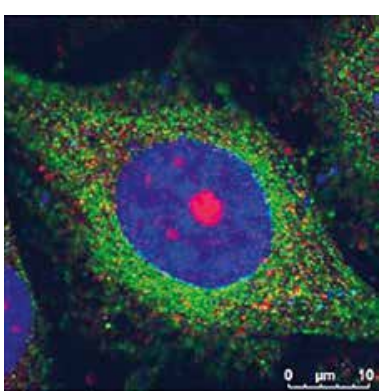

Fig. 2. Differential expression of protein phosphatases 1 gamma 2 (PP1 2 2) in whole cell (WC), nuclear (NC) and cytosolic (CYT) fractions of cervical cancer HeLa cell. A - western blot showing PP1 $\gamma 2$ protein expression in the WC, NC and CYT fractions of HeLa cells, B - densitometry analysis of the western blot confirmed the differential expression of PP1 $\gamma 2$. $\beta$-actin and Histone- 3 were used as a loading control for the cytoplasmic and nuclear fractions, respectively. These results are presented as the mean $\pm \mathrm{SEM}$. of 3 independent experiments, ${ }^{* *} p<0.01$; as compared to WC, C - sub-cellular localization of PP1 $\gamma 2$ in HeLa cells showed predominant expression of the PP1 $\gamma 2(r)$ in the nuclear region of the cell, while discrete patches were also observed in the cytoplasm. Blue (b) represents nuclear stain DAPI and green (g) staining shows tubulin expression. Zoom represents $4 x$ magnification of the original. All images were captured using an oil immersion $63 x$ objective lens

designed to assess the phosphorylation level of GSK3 $\beta$ after the silencing of the expression of PP1 $\gamma 2$ through siRNA. Hence 2 siRNAs, including Si1 \& Si2, were designed specifically to silence the expression of PP1 12 . Western blotting of these transfected cells revealed that siRNA-mediated silencing of PP1 2 downregulated the expression of PP1 $\gamma 2$ and increased the phosphorylation of GSK3 $\beta[19,20]$. Densitometric analysis of the data further confirmed the increased phosphorylation of GSK3 $\beta$ within $24 \mathrm{hrs}$, which was significant within 48 hrs for Si2 treated cells (Fig. 5 A, B).

\section{Discussion}

Various isoforms of PP play a pivotal role in cell cycle regulation [21] and cytokinesis [21] while being responsible for mitotic exit. Two isoforms of PP1 $\gamma$ are known to express by the alternate splicing of the PPP1CC gene. PP1 1 1 is expressed ubiquitously and has been explored intricately in cell cycle regulation [10]; however, PP1 $\gamma 2$ as a testis-specific isoform [9] has not been investigated extensivley with respect to cell cycle regulation and tumourigenesis. The present study reports the expression of PP1 22 RNA (Fig. 1 A) as well as protein (Fig. 1 B) in cervical cancer HeLa cells. Flow cytometry analysis revealed prominent subcellular expression of PP1 $\gamma 2$ in permeabilized HeLa cells, whereas no signal was observed in the unpermeabilized HeLa cells depicting no cell surface expression of PP1 $\gamma 2$ in HeLa cells (Fig. 1 C).

Earlier reports revealed enriched PP1 activity in the nucleus of the cell $[22,23]$. To assess the subcellular distribution of PP1 $\gamma 2$ in HeLa cells, the protein was extracted from whole-cell, nuclear and cytoplasmic fractions and subjected to western blotting; these results demonstrated predominant expression of PP1 $\gamma 2$ in the nucleus as compared to the discrete distribution in the cytoplasmic fraction (Fig. 2 A, B). Furthermore, immunofluorescence studies also localized PP1 $\gamma 2$ to the nucleus of mononuclear interphase HeLa cells (Fig. 2 C) and relocated to the spindle poles during the mitotic phase. In the dividing cells, PP1 $\gamma 2$ 

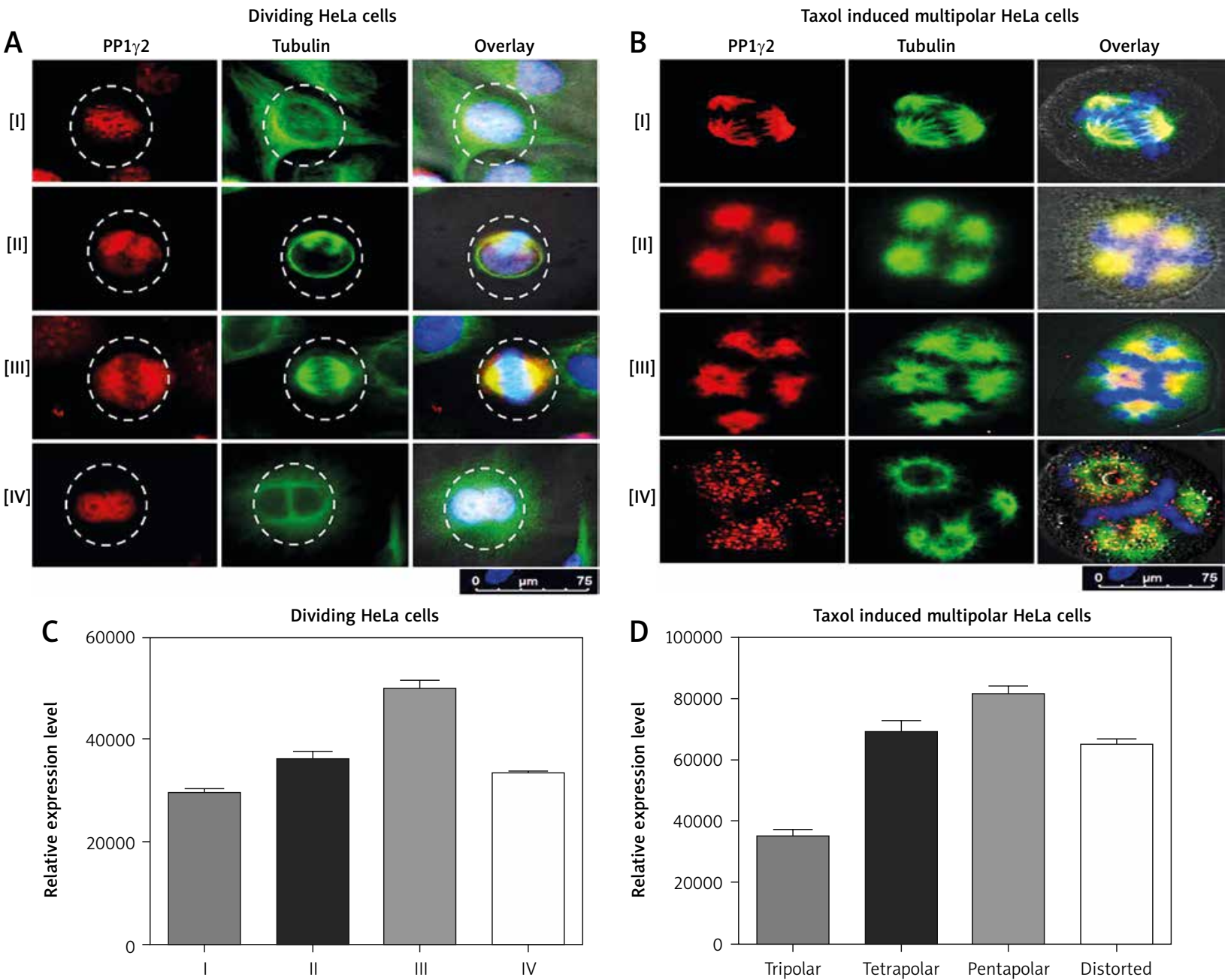

Fig. 3. Spatio-temporal distribution of protein phosphatases 1 gamma 2 (PP1 $\gamma 2$ ) (Red) and tubulin (green) in HeLa cells. A - in dividing HeLa cells: PP1 $\gamma 2$ is localized to the nucleus of the mononuclear cells, whereas tubulin localized in the cytosol (I). PP1 $\gamma 2$ started redistributing to the poles at the initiation of the cell division, where tubulin localized to both the spindle poles as well as to the cell periphery (II). Localization of PP1 $\gamma 2$ merged completely with tubulin to the poles of the bipolar cells in mitotic phase (III). After complete cell division, PP1 22 re-localized again back to the nucleus, whereas tubulin remained in the cytosol only (IV), B - in Taxol-induced multipolar HeLa cells, increased expression of PP1 $\gamma 2$ merged completely with tubulin on multiple spindle poles of all the multipolar cells, including tripolar (I), tetrapolar (II), and pentapolar cells (III). Punctuated staining of PP1 22 was observed in the vicinity of the distorted chromosomal apparatus, where tubulin remained located in the cytosol itself (IV). Densitometry analysis through ImageJ software showing relative expression of PP1 $\gamma 2$ in (C) single dividing HeLa cells, D - in Taxol-induced multipolar HeLa cells
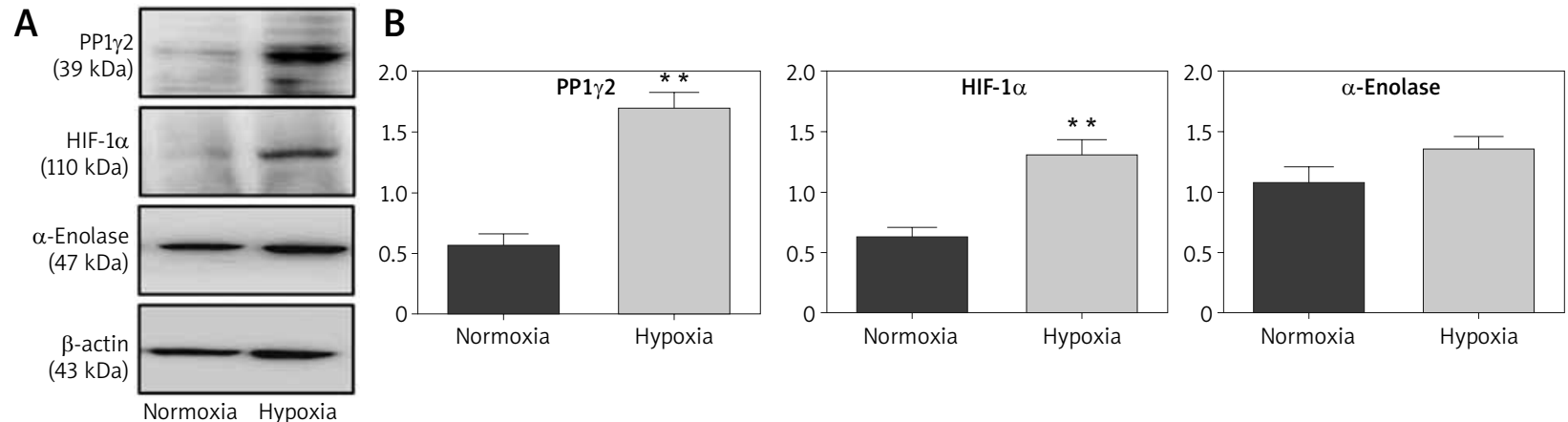

Fig. 4. Upregulated expression of protein phosphatases 1 gamma 2 (PP1 2 2) in hypoxic condition of cervical cancer HeLa cells. A - western blots showing the upregulation of PP1 $\gamma 2$, HIF1- $\alpha$, and $\alpha$-enolase in hypoxic environment of HeLa cells, B - densitometry analysis confirmed these results with the statistical significance for PP1 $\gamma 2$ and HIF1- $\alpha$, whereas upregulation of $\alpha$-Enolase was not statistically significant. At least 3 independent experiments were done and data was normalized with $\beta$-actin, the results are presented as mean \pm SEM ${ }^{* \star} p<0.01$ 

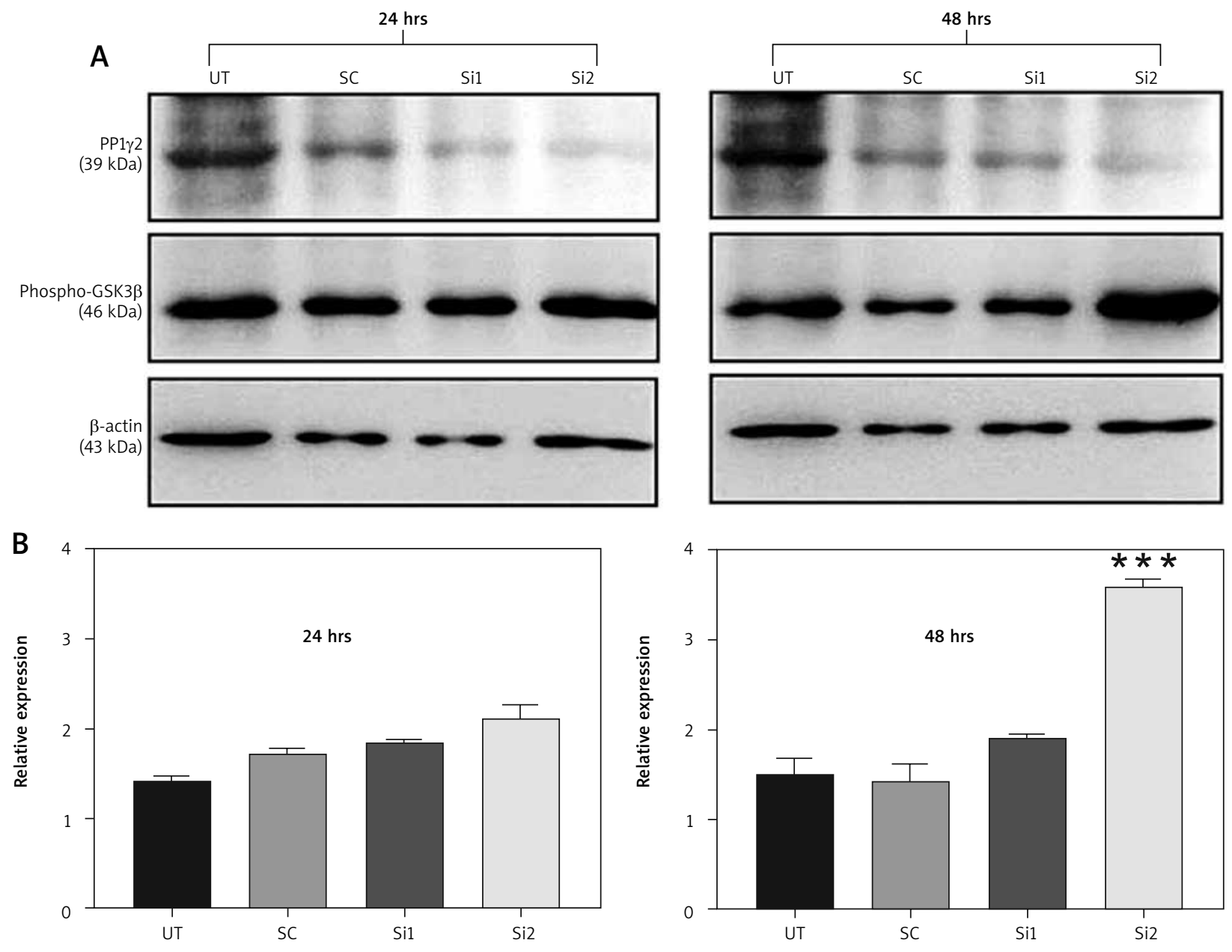

Fig. 5. Protein phosphatases 1 gamma 2 (PP1 $\gamma 2$ ) silencing increased the phosphorylation of GSK3ß. A - PP1 1 2-siRNA treatment in HeLa cells downregulated the expression of PP1 $\gamma 2(\sim 39 \mathrm{kDa})$ and increased phosphorylation of GSK3 $\beta(\sim 46 \mathrm{kDa}), \mathbf{B}-$ densitometric analysis of PP1 $\gamma 2$-silenced cells further confirmed increased phosphorylation of GSK3 $\beta$ within 24 hrs, which was quite significant within 48 hrs of Si-1 treatment. At least 3 independent experiments were done, and data of the study are presented as mean $\pm \mathrm{SEM},{ }^{\star * *} p<0.001$, compared to UT

redistributed to the spindle poles and appeared to be more condensed at the poles, completely merged with the tubulin localization. At the same time, as soon as cell division was completed, PP1 $\gamma 2$ localized back to the nucleus of the divided cells (Fig. 3 A), whereas tubulin localization was seen in the cytosol only. Hence, these observations can speculate that PP1 22 may interact with the spindle organizing apparatus transiently and may regulate the tubulin for spindle pole formation.

To confirm the spatio-temporal redistribution of PP1 $\gamma 2$ in dividing cells, multipolar HeLa cells were induced by Taxol. As Taxol inhibits tubulin depolymerization and arrests the cells in the G2/M phase, and accelerates apoptosis [6], these cells showed intense staining of PP1 $\gamma 2$ at the multiple spindle poles (Fig. 3 B) and overlaid completely with the tubulin staining. Because these multipolar cells become polyploid and eventually go through apoptosis, intense localization of PP1 $\gamma 2$ on the poles indicates its possible role in regulating spindle formation and cell division.

To understand the effect of the hypoxic tumour microenvironment on PP1 $\gamma 2$, its expression level was observed under hypoxic conditions. Hypoxia is a prominent characteristic of cancerous cells governed by hypoxia-inducible factor- $1 \alpha$ (HIF-1 $\alpha$ ), a key transcription factor, and regulates many downstream targets [24]. In cancerous cells, the hypoxic condition has been associated with radio- and chemoresistance and inhibits apoptosis, which alters EMT cell signalling and genomic stability and induces downstream cell proliferation. Observations and findings from the present data demonstrated an upregulated level of PP1 $\gamma 2$ (Fig. 4). Hence, it might be associated with a defensive role against hypoxia-induced stress, which promotes cancer cell survival under hypoxic conditions and thus leads to poor prognosis.

Further to assess the functional role of PP1 $\gamma 2$ with downstream effectors, siRNA-mediated silencing of PP1 22 in HeLa cells showed increased phosphorylation of GSK3 $\beta$ (Fig. 5). The role of GSK3 $\beta$ has been reflected in the cell cycle regulation of cancerous cells[18]. Simultaneously, the interaction of PP1 $\gamma 2$ with GSK3 $\beta$ has been reported in spermatocytes [25], where it regulates spermatogenesis. GSK-3 $\beta$ is well documented to participate in a complex array of critical cellular processes. During mitosis, a phos-

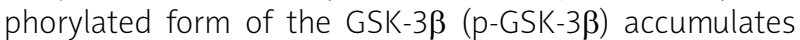
at centrosomes and spindle poles. GSK-3 $\beta$ is a direct substrate for PP1 $\gamma 2$; where GSK-3 $\beta$ is being dephosphorylated 
through PP1 $\gamma 2$ and produces an active form of GSK-3 3 . Earlier studies have also predicted the role of active GSK-3 $\beta$ (dephosphorylated form) in the regulation of microtubule dynamics [26].

In the present study, silencing of PP1 $\gamma 2$ made it unavailable to dephosphorylate GSK-3 $\beta$ and led to the increased

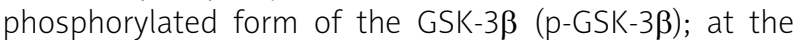
same time, total GSK-3 $\beta$ did not show much difference. Interestingly, the silencing of PP1 22 through siRNA upregulates the phosphorylation of GSK3 $\beta$ and hence also affects the downstream signalling. Therefore, silencing of PP1 $\gamma 2$ may indirectly target GSK3 $\beta$ and it remains inactive in the phosphorylated form, which regulates the downstream microtubule dynamics.

\section{Conclusions}

Tumour cells frequently express genes that are normally restricted to the testis, known as cancer/testis antigens. Similarly, so far, expression of PP1 $\gamma 2$ had been reported as a testis-specific isoform; however, for the first time, the present study demonstrated a spatio-temporal redistribution of PP1 $\gamma 2$ during the cell cycle of HeLa cells. Silencing of PP1 $\gamma 2$ through siRNA also demonstrated the regulation of GSK3 $\beta$ phosphorylation, which is a well-known regulator of the cell cycle; hence this study verified PP1 2 as an upstream player, where PP1 22 modulation plays an important role during cell cycle regulation.

\section{Acknowledgements}

Authors acknowledge Indian Council of Scientific \& Industrial Research, New Delhi for the project funding, and Indian Council of Medical Research, New Delhi for providing fellowship to Saurabh Kumar Agnihotri. Dr. A L Vishwakarma from Sophisticated Analytical Instruments Facility, CSIR-Central Drug Research Institute Lucknow is also thankfully acknowledged for the flow cytometry facility.

The authors declare no conflicts of interest.

\section{References}

1. Hofmann TG, Möller A, Sirma H, et al. Regulation of p53 activity by its interaction with homeodomain-interacting protein kinase-2. Nat Cell Biol 2002; 4: 1-10.

2. Fernandez A, Brautigan DL, Lamb NJC. Protein phosphatase type 1 in mammalian cell mitosis: chromosomal localization and involvement in mitotic exit. J Cell Biol 1992; 116: 1421-1430.

3. Olsen JV, Blagoev B, Gnad F, et al. Global, in vivo, and site-specific phosphorylation dynamics in signaling networks. Cell 2006; 127: 635-648.

4. Moorhead GBG, Trinkle-Mulcahy L, Ulke-Lemée A. Emerging roles of nuclear protein phosphatases. Nat Rev Mol Cell Biol 2007; 8: 234-244.

5. Andreassen PR, Lacroix FB, Villa-Moruzzi E, Margolis RL. Differential subcellular localization of protein phosphatase- $1 \alpha, \gamma 1$, and $\delta$ isoforms during both interphase and mitosis in mammalian cells. J Cell Biol 1998; 141: 1207-1215.

6. Trinkle-Mulcahy L, Sleeman JE, Lamond Al. Dynamic targeting of protein phosphatase 1 within the nuclei of living mammalian cells. J Cell Sci 2001; 114: 4219-4228.

7. Wu JQ, Guo JY, Tang W, et al. PP1-mediated dephosphorylation of phosphoproteins at mitotic exit is controlled by inhibitor-1 and PP1 phosphorylation. Nat Cell Biol 2009; 11: 644-651.
8. Schmitz MHA, Held $M$, Janssens $V$, et al. Live-cell imaging RNAi screen identifies PP2A-B55 $\alpha$ and importin- $\beta 21$ as key mitotic exit regulators in human cells. Nat Cell Biol 2010; 12: 886-893.

9. Chakrabarti R, Kline D, Lu J, Orth J, Pilder S, Vijayaraghavan S. Analysis of Ppp1cc-null mice suggests a role for PP1 gamma 2 in sperm morphogenesis. Biol Reprod 2007; 76: 992-1001.

10. Trinkle-Mulcahy L, Andrews PD, Wickramasinghe S, et al. Timelapse imaging reveals dynamic relocalization of PP1 $\gamma$ throughout the mammalian cell cycle. Mol Biol Cell 2003; 14: 107-117.

11. Cheng L, Pilder S, Nairn AC, Ramdas S, Vijayaraghavan S. PP1 $\gamma 2$ and PPP1R11 are parts of a multimeric complex in developing testicular germ cells in which their steady state levels are reciprocally related. PLoS One 2009; 4: e4861.

12. Schmutz S, Wendt A, Schnell A, Kramer A, Mansuy IM, Albrecht U. Protein phosphatase 1 (PP1) is a post-translational regulator of the mammalian circadian clock. PLoS One 2011; 6: e21325.

13. Bruggeman W, Koster J, Lodder P, Repping S, Hamer G. Massive expression of germ cell-specific genes is a hallmark of cancer and a potential target for novel treatment development. Oncogene 2018; 37: 5694-5700

14. Chomczynski P, Mackey K. Substitution of chloroform by bromochloropropane in the single-step method of RNA isolation. Anal Biochem 1995; 225: 163-164.

15. Bradford M. A rapid and sensitive method for the quantitation of microgram quantities of protein utilizing the principle of protein-dye binding. Anal Biochem 1976; 72: 248-254.

16. Billadeau DD. Primers on molecular pathways: the glycogen synthase kinase-3ß. Pancreatology 2007; 7: 398-402.

17. Seldin DC, Landesman-Bollag E, Farago M, Currier N, Lou D, Dominguez I. CK2 as a positive regulator of Wnt signalling and tumourigenesis. Mol Cell Biochem 2005; 274: 63-67.

18. Wakefield G, Stephens DJ, Tavaré JM. A role for glycogen synthase kinase-3 in mitotic spindle dynamics and chromosome alignment. J Cell Sci 2003; 116: 637-646.

19. Mochida S, Hunt T. Protein phosphatases and their regulation in the control of mitosis. EMBO Rep 2012; 13: 197-203.

20. Kim HS, Fernandes G, Lee CW. Protein phosphatases involved in regulating mitosis: facts and hypotheses. Mol Cells 2016; 39: 654-662.

21. Rosa AM, Dabas N, Byrnes DM, Eller MS, Grichnik JM. Germ cell proteins in melanoma: prognosis, diagnosis, treatment, and theories on expression. J Skin Cancer 2012; 2012: 1-8.

22. Vagnarelli P, Hudson DF, Ribeiro SA, et al. Condensin and RepoMan-PP1 co-operate in the regulation of chromosome architecture during mitosis. Nat Cell Biol 2006; 8: 1133-1142.

23. Kuret J, Bell H, Cohen P. Identification of high levels of protein phosphatase-1 in rat liver nuclei. FEBS Lett 1986; 203: 197-202.

24. Graeber TG, Osmanian C, Jacks T, et al. Hypoxia-mediated selection of cells with diminished apoptotic potential in solid tumours. Nature 1996; 379: 88-91.

25. Chun YS, Shima H, Nagasaki K, Sugimura T, Nagao M. PP1 $\gamma 2$, a testis-specific protein-serine/threonine-phosphatase type 1 catalytic subunit, is associated with a protein having high sequence homology with the 78-kDa glucose-regulated protein, a member of the 70-kDa heat shock protein family. Proc Natl Acad Sci U. S. A. 1994; 91: 3319-3323.

26. Lovestone S, Hartley CL, Pearce J, Anderton BH. Phosphorylation of tau by glycogen synthase kinase- $3 \beta$ in intact mammalian cells: the effects on the organization and stability of microtubules. Neurosci 1996; 73: 1145-1157.

\section{Address for correspondence}

\section{Monika Sachdev PhD}

Endocrinology Division

CSIR-Central Drug Research Institute

Lucknow 226 031, India

e-mail: monika@cdri.res.in

Submitted: 22.03 .2021

Accepted: 13.05.2021 